\title{
A LEMMA ON THE DIFFERENCE QUOTIENTS
}

\author{
Risto Korhonen, Kazuya Tohge, Yueyang Zhang and Jianhua Zheng \\ University of Eastern Finland, Department of Physics and Mathematics \\ P. O. Box 111, FI-80101 Joensuu, Finland; risto.korhonen@uef.fi \\ Kanazawa University, College of Science and Engineering \\ Kakuma-machi, Kanazawa, 920-1192, Japan; tohge@se.kanazawa-u.ac.jp
}

University of Science and Technology Beijing, School of Mathematics and Physics

No. 30 Xueyuan Road, Haidian, Beijing, 100083, P. R. China; zhangyueyang@ustb.edu.cn

Tsinghua University, Department of Mathematical Sciences

Beijing 100084, P. R. China; zheng-jh@mail.tsinghua.edu.cn

\begin{abstract}
First, we are concerned with a lemma on the difference quotients due to Halburd, Korhonen and Tohge. We show that for meromorphic functions whose deficiency is origin dependent the exceptional set associated with this lemma is of infinite linear measure. In particular, for such entire functions in this set there is an infinite sequence $\left\{r_{n}\right\}$ such that $m\left(r_{n}, f(z+c) / f(z)\right) \neq$ $o\left(T\left(r_{n}, f\right)\right)$ for all $r_{n}$. Then we extend this lemma to the case of meromorphic functions $f(z)$ such that $\log T(r, f) \leq a r /(\log r)^{2+\nu}, a, \nu>0$, for all sufficiently large $r$, by using a new Borel type growth lemma. Second, we give a discrete version of this Borel type growth lemma and use it to provide an extension of Halburd's result on first order discrete equations of Malmquist type.
\end{abstract}

\section{Introduction}

The lemma on the logarithmic derivatives is one of the key results needed in proving Nevanlinna's second main theorem [25], as well as an important tool in analyzing value distribution of entire and meromorphic solutions of differential equations [22]. Nevanlinna theoretic approach by Ablowitz, Halburd and Herbst [1] to study difference Painlevé equations leads to a need of finding extensions of value distribution theory for difference operators. A lemma on difference quotients for finite order meromorphic functions was introduced in two independent studies, by Halburd and the first author [16, 17], and by Chiang and Feng [8]. The lemma on difference quotients was later on extended to include meromorphic functions $f(z)$ of hyper-order less than one by Halburd and the first and the second author [19] and recently to include meromorphic functions with hyper-order equal to 1 and with minimal type by Zheng and Korhonen [31]. For a non-constant meromorphic function $f(z)$ on the complex plane, the order $\sigma$ and the hyper-order $\varsigma$ of $f$ are defined, respectively, as

$$
\sigma=\sigma(f)=\limsup _{r \rightarrow \infty} \frac{\log T(r, f)}{\log r}
$$

https://doi.org/10.5186/aasfm.2020.4521

2010 Mathematics Subject Classification: Primary 30D35; Secondary 30D30.

Key words: Difference quotient, growth of meromorphic functions, Diophantine integrability, algebraic entropy.

The first author is supported in part by the Academy of Finland grant (\#286877) and (\#268009), and the second author is supported by the JSPS KAKENHI Grant Number JP16K05194, the third author is supported by the China Scholarship Council (CSC) and the Fundamental Research Funds for the Central Universities (FRF-TP-19-055A1), the fourth author is supported by the grant (no. 11571193) of NSF of China. 
and

$$
\varsigma=\varsigma(f)=\limsup _{r \rightarrow \infty} \frac{\log \log T(r, f)}{\log r},
$$

where $T(r, f)$ is the Nevanlinna characteristic. The lemma on difference quotients for meromorphic functions of hyper-order $\varsigma<1$ introduced in [19] takes the form

$$
m\left(r, \frac{f(z+c)}{f(z)}\right)=o\left(\frac{T(r, f)}{r^{1-\varsigma-\varepsilon}}\right),
$$

where $\varepsilon>0, c \in \mathbf{C} \backslash\{0\}$ and $r \rightarrow \infty$ outside of a set of finite logarithmic measure. A set $E$ is said to have finite logarithmic measure if $\int_{E \cap[1, \infty)} d r / r<\infty$. The version of (1.1) relies on an independent asymptotic estimate for $T(r, f)$ [19]. The estimate by Chiang and Feng [8] for meromorphic functions of finite order $\sigma$ can be written in the form

$$
m\left(r, \frac{f(z+c)}{f(z)}\right)=O\left(r^{\sigma-1+\varepsilon}\right)
$$

where $r \rightarrow \infty$ without an exceptional set. Chiang and Feng [9, 10] and, independently, Bergweiler and Langley [5] have obtained Wiman-Valiron type estimates for difference quotients in the case of order $<1$ meromorphic functions. These results extend a Wiman-Valiron method for differences due to Ishizaki and Yanagihara [21]. Recently, Chiang and Feng [11] obtained the Askey-Wilson logarithmic difference estimate for meromorphic functions of finite logarithmic order, and Cheng and Chiang [6] proved a lemma on the logarithmic Wilson differences for finite order meromorphic functions.

Zheng and Korhonen [31] prove the necessity of the appearance of the exceptional set in (1.1) by providing an example of entire function of finite order such that $m(r, f(z+c) / f(z)) \neq o(T(r, f))$ for all $r$ in a set $E$ of infinite linear measure (i.e. $\left.\int_{E \cap[1, \infty)} d r=\infty\right)$ and of positive finite logarithmic measure. In Section 2 we further study the behavior of $m(r, f(z+c) / f(z))$ in the exceptional set in (1.1) for a class of meromorphic functions whose deficiency is dependent on the choice of origin. For such meromorphic functions the error term $O\left(r^{\sigma-1+\varepsilon}\right)$ on the right hand side of (1.2) is bigger than the characteristic $T(r, f)$ for a large part of the positive real line. We show that for such meromorphic functions the exceptional set in the estimate (1.1) is of infinite linear measure and in the entire function case there is an infinite sequence $\left\{r_{n}\right\}$ in the exceptional set such that for all $r_{n}$,

$$
m\left(r_{n}, \frac{f(z+c)}{f(z)}\right) \neq o\left(T\left(r_{n}, f\right)\right) .
$$

In Section 3 we extend the lemma on difference quotients to a slightly more general case where $f$ is a meromorphic function such that $\log T(r, f) \leq a r /(\log r)^{2+\nu}, a, \nu>$ 0 , for all $r$ sufficiently large by showing that for such functions

$$
m\left(r, \frac{f(z+c)}{f(z)}\right)=o\left(\frac{T(r, f)}{(\log r)^{\nu-\varepsilon}}\right),
$$

where $r \rightarrow \infty$ outside of a set of finite logarithmic measure. A key tool in the proof of this extension is a new Borel type growth lemma. A purely discrete form of the Borel lemma is given in Section 4, which extends a result due to Al-Ghassani and Halburd [2]. Finally, we obtain a Malmquist type theorem for first order discrete equations in Section 5 as an application of the discrete Borel lemma. 


\section{Growth of the difference quotients in the exceptional set}

For a meromorphic function of finite order $\sigma$, we can also obtain the error term $O\left(r^{\sigma-1+\varepsilon}\right)$ in (1.2) from the estimates given in [16, Lemma 2.3] or [19, Lemma 8.2] by suitably choosing the coefficients there. Zheng and Korhonen [31] have given an alternative proof of (1.2) using a different approach. Unlike in the estimate (1.1), when $\sigma \geq 1$ the growth relation between the error term $O\left(r^{\sigma-1+\varepsilon}\right)$ and $T(r, f)$ is unclear apart from a set of infinite logarithmic measure [18, Corollary 3.3]. Consider a meromorphic function whose lower order is a finite number $\lambda$ which is defined by

$$
\lambda=\lambda(f)=\liminf _{r \rightarrow \infty} \frac{\log T(r, f)}{\log r} .
$$

Let $\varepsilon>0$ be given. Then the above definition implies that $T(r, f) \leq r^{\lambda+\varepsilon}$ holds for all $r$ in a set of infinite logarithmic measure [13]. We choose an infinite sequence $\left\{r_{n}\right\}$ such that $r_{n+1} \geq 2 r_{n}, r_{1}>1$ and that

$$
\lim _{n \rightarrow \infty} \frac{\log T\left(r_{n}, f\right)}{\log r_{n}}=\lambda
$$

For the given $\varepsilon>0$, it follows that there is an integer $N_{0}$ such that $T\left(r_{n}, f\right) \leq r_{n}^{\lambda+\varepsilon}$ for all $n>N_{0}$. Now for all $r \in\left[r_{n} / 2, r_{n}\right], n>N_{0}$ we have

$$
T(r, f) \leq T\left(r_{n}, f\right) \leq r_{n}^{\lambda+\varepsilon}=\left(\frac{r_{n}}{r}\right)^{\lambda+\varepsilon} \cdot r^{\lambda+\varepsilon} \leq\left(\frac{r_{n}}{r_{n} / 2}\right)^{\lambda+\varepsilon} \cdot r^{\lambda+\varepsilon}=2^{\lambda+\varepsilon} \cdot r^{\lambda+\varepsilon} .
$$

Obviously, the set $E=\cup_{n=1}^{\infty}\left[r_{n} / 2, r_{n}\right]$ has infinite logarithmic measure. Thus, if $\sigma-1 \geq \lambda$, then there is a constant $a>0$ such that $r^{\sigma-1+\varepsilon} \geq r^{\lambda+\varepsilon} \geq a T(r, f)$ for all $r \in E$. Recall that the deficiency $d(0, f)$ of a meromorphic function $f$ for the value 0 is defined as

$$
d(0, f):=1-\limsup _{r \rightarrow \infty} \frac{N(r, 1 / f)}{T(r, f)}=\liminf _{r \rightarrow \infty} \frac{m(r, 1 / f)}{T(r, f)} .
$$

Valiron [28] (see also [26, p. 271]) proved the following result concerning the dependence on the choice of origin of the deficiency of any meromorphic function $f$ : If the characteristic function $T(r, f)$ of $f$ satisfies

$$
\lim _{r \rightarrow \infty} \frac{T(r+1, f)}{T(r, f)}=1
$$

then the deficiency of $f$ is independent on the choice of origin, that is, $d(0, f(z))=$ $d(0, f(z+c))$ for any nonzero constant $c$. Moreover, if $f$ has finite order $\sigma$ and lower order $\lambda$, then the condition (2.1) can also be replaced by $\sigma-\lambda<1$. Thus we have

Theorem 2.1. Let $f$ be a meromorphic function of finite order $\sigma$ and $\varepsilon>0$. If $d(0, f(z)) \neq d(0, f(z+c))$ for some nonzero constant $c$, then $O\left(r^{\sigma-1+\varepsilon}\right) \neq o(T(r, f))$ for all $r$ in a set of infinite logarithmic measure.

Chiang and Luo [12, p. 455] have pointed out that the estimate (1.2) together with [8, Theorem 2.1] implies the finite order case of Valiron's result above since when $\sigma-\lambda<1$ the error term $O\left(r^{\sigma-1+\varepsilon}\right)=o(T(r, f))$ for all $r \rightarrow \infty$, but we also need to know what happens to $O\left(r^{\sigma-1+\varepsilon}\right)$ when the deficiency is dependent on the choice of origin.

Theorem 2.1 shows that if we remove the exceptional set in the estimate (1.1) and formulate the error term in terms of the order of a meromorphic function, then the error term can grow faster than $T(r, f)$ on a much larger part of the positive 
real line. For meromorphic functions whose deficiency is dependent on the choice of origin, we may suppose without loss of generality that $d(0, f(z))>d(0, f(z+c))$, where $c \neq 0$ is a constant. For these functions, we prove the following

Theorem 2.2. Let $f$ be a meromorphic function of hyper-order less than 1 and $d(0, f(z))>d(0, f(z+c))$ for some $c \neq 0$. Then the exceptional set in (1.1) is of infinite linear measure. In particular, if $f$ is entire, then for any infinite sequence $\left\{r_{n}\right\}$ such that

$$
\lim _{n \rightarrow \infty} \frac{m\left(r_{n}, 1 / f(z+c)\right)}{T\left(r_{n}, f(z+c)\right)}=d(0, f(z+c))
$$

we have

$$
\liminf _{n \rightarrow \infty} \frac{m\left(r_{n}, f(z+c) / f(z)\right)}{T\left(r_{n}, f(z)\right)} \geq \frac{d(0, f(z))-d(0, f(z+c))}{1+d(0, f(z+c))} .
$$

Theorem 2.2 shows that for meromorphic functions of hyper-order $<1$ whose deficiency is dependent on the choice of origin, the exceptional set out of consideration in the proof of (1.1) in [19] is of infinite linear measure. Obviously, the infinite sequence $\left\{r_{n}\right\}$ is in the exceptional set for all sufficiently large $r_{n}$. It is still unknown whether $m(r, f(z+c) / f(z)) \neq o(T(r, f))$ holds on a set of infinite linear measure for any meromorphic function $f$ whose deficiency is dependent on the choice of origin.

To prove Theorem 2.2, we first prove the following Proposition 2.3, which is concerned with a counterpart of condition (2.1) and complements the result due to Valiron [28] (see also [26, p. 271]) mentioned above.

Proposition 2.3. Let $f$ be a non-constant meromorphic function. If $d(0, f(z))>$ $d(0, f(z+c))$ for some $c \neq 0$, then there exists a constant $1<C<\infty$ such that

$$
T(r+|c|, f) \geq C T(r, f)
$$

for all $r$ in a set with infinite linear measure.

Proof. For simplicity, denote $d_{1}=d(0, f(z))$ and $d_{2}=d(0, f(z+c))$. Then $0 \leq d_{2}<d_{1} \leq 1$. Recall the following double inequalities from [13, p. 47] (see also $[1,30])$,

$$
\begin{aligned}
(1+o(1)) T(r-|c|, f(z)) & \leq T(r, f(z+c)) \leq(1+o(1)) T(r+|c|, f(z)) \\
(1+o(1)) N(r-|c|, 1 / f(z)) & \leq N(r, 1 / f(z+c)) \leq(1+o(1)) N(r+|c|, 1 / f(z)),
\end{aligned}
$$

where $r \rightarrow \infty$ and $N(r, 1 / f)$ is the integrated counting function for zeros of $f$. Then we have

$$
\frac{N(r, 1 / f(z+c))}{T(r, f(z+c))} \leq(1+o(1)) \cdot \frac{N(r+|c|, 1 / f(z))}{T(r+|c|, f(z))} \cdot \frac{T(r+|c|, f(z))}{T(r-|c|, f(z))} .
$$

By letting $r \rightarrow \infty$ and taking the superior limit on both sides of the above inequality, we get

$$
\limsup _{r \rightarrow \infty} \frac{T(r+|c|, f)}{T(r-|c|, f)}=\infty
$$

in the case $d_{1}=1$ and

$$
\limsup _{r \rightarrow \infty} \frac{T(r+|c|, f)}{T(r-|c|, f)} \geq \frac{1-d_{2}}{1-d_{1}}
$$

in the case $d_{1}<1$. Let $C$ be a real constant such that $1<C<\infty$ when $d_{1}=1$ and $1<C^{3}<\left(1-d_{2}\right) /\left(1-d_{1}\right)$ when $d_{1}<1$. Define the set

$$
E=\{r: T(r+|c|, f) \geq C T(r, f)\} \text {. }
$$


We claim that this set has infinite linear measure. Otherwise, the inequality

$$
T(r+|c|, f) \leq C T(r, f)
$$

holds for all $r$ outside an exceptional set with finite linear measure. Recall the following lemma from [18, Lemma 3.1]: Let $\mu$ be a positive, strictly increasing differentiable function of $r$ defined on $\left(r_{0}, \infty\right)$ for some $r_{0}$ and let $g_{1}(r)$ and $g_{2}(r)$ be two non-decreasing functions for all $r_{0}<r<\infty$ such that $g_{1}(r) \leq g_{2}(r)$ for all $r \in\left(r_{0}, \infty\right) \backslash E$, where the exceptional set $E$ satisfies

$$
\int_{t \in E \cap\left[r_{0}, \infty\right)} d \mu(t)<\infty
$$

Then, for a given $\epsilon>0$, there is an $\hat{r} \geq r_{0}$ such that $g_{1}(r) \leq g_{2}(s(r))$ for all $r \geq \hat{r}$, where $s(r)=\mu^{-1}(\mu(r)+\epsilon)$. An application of this lemma with $\mu(r)=r$ to $(2.7)$ yields that, for a given $\epsilon$ such that $0<3 \epsilon \leq|c|$, there is a large enough $r_{0}$ such that for all $r \in\left[r_{0}, \infty\right)$,

$$
T(r+|c|, f) \leq C T(r+\epsilon, f) .
$$

Thus the superior limit of $T(r+|c|, f) / T(r+\epsilon, f)$, as $r$ approaches $\infty$, is at most $C$. But then

$$
\begin{aligned}
\limsup _{r \rightarrow \infty} \frac{T(r+|c|, f)}{T(r-|c|, f)} & \leq \limsup _{r \rightarrow \infty} \frac{T(r+|c|, f)}{T(r+\epsilon, f)} \cdot \frac{T(r+\epsilon, f)}{T(r-|c|+2 \epsilon, f)} \cdot \frac{T(r-|c|+2 \epsilon, f)}{T(r-|c|, f)} \\
& \leq C^{3},
\end{aligned}
$$

a contradiction to (2.4) or (2.5). Hence the set defined in (2.6) must be of infinite linear measure and our assertion follows.

Proof of Theorem 2.2. To obtain the estimate (1.1), the Borel type growth lemma [19, Lemma 8.3] which holds for all $r$ outside of a set of finite logarithmic measure is needed. Proposition 2.3 implies that this set is of infinite linear measure since the constant $C$ in Proposition 2.3 satisfies $C \geq 1+\eta / r^{\delta}$ when $r$ is sufficiently large. However, the estimates in [19, Lemma 8.2] and in [19, Lemma 8.3] are independent to each other and the exceptional set in [19, Lemma 8.3] will not appear in (1.1) whenever $T(r, f)$ satisfies

$$
\limsup _{r \rightarrow \infty} \frac{T(r+|c|, f)}{T(r, f)}<\infty
$$

From the proof of Proposition 2.3 we see that this case is possible when $d(0, f(z))<1$ under our assumptions. So we need to consider the exceptional set which is defined as:

$$
E_{1}=\left\{r: T\left(r+|c|+\frac{r+|c|}{\xi(T(r+|c|, f))}\right) \geq C T(r+|c|, f)\right\},
$$

where, as in the proof of [16, Theorem 2.1] and [19, Theorem 5.1], the function $\xi(x)$ is chosen as $\xi(x)=x^{\varepsilon / 2}$ when $f$ is of finite order and $\xi(x)=(\log x)^{1+\varepsilon / 3}$ when $f$ is of infinite order respectively and $\varepsilon>0$ satisfies $(\varsigma+\varepsilon / 3)(1+\varepsilon / 3)<1$.

For simplicity, denote $d_{1}=d(0, f(z))$ and $d_{2}=d(0, f(z+c))$. By assumption we have $d_{1}>d_{2}$. It follows from Proposition 2.3 that for some finite constant $C>1$ the set $E_{2}=\{r: T(r+2|c|, f) \geq C T(r+|c|, f)\}$ is of infinite linear measure. From the definition of $E_{1}$ in (2.8), it is seen that there is a sufficiently large $r_{0}$ such that 
$E_{2} \cap\left[r_{0}, \infty\right) \subseteq E_{1} \cap\left[r_{0}, \infty\right)$. Thus the exceptional set in (1.1) must be of infinite linear measure. Moreover, if $f$ is an entire function, then we deduce that

$$
\begin{aligned}
T(r, f(z+c)) & =m(r, f(z+c)) \leq m(r, f(z))+m\left(r, \frac{f(z+c)}{f(z)}\right) \\
& =T(r, f(z))+m\left(r, \frac{f(z+c)}{f(z)}\right) .
\end{aligned}
$$

Note that $d_{1}>d_{2}$, and let $\left\{r_{n}\right\}$ be an arbitrary sequence such that

$$
\lim _{n \rightarrow \infty} \frac{m\left(r_{n}, 1 / f(z+c)\right)}{T\left(r_{n}, f(z+c)\right)}=d_{2}
$$

Then, for any $\varepsilon$ satisfying $0<2 \varepsilon<d_{1}-d_{2}$, we have

$$
m\left(r_{n}, \frac{1}{f(z+c)}\right) \leq\left(d_{2}+\varepsilon\right) T\left(r_{n}, f(z+c)\right)
$$

for all sufficiently large $r_{n}$. By (2.9) and (2.10), we get

$$
m\left(r_{n}, \frac{1}{f(z+c)}\right) \leq\left(d_{2}+\varepsilon\right) T\left(r_{n}, f(z)\right)+\left(d_{2}+\varepsilon\right) m\left(r_{n}, \frac{f(z+c)}{f(z)}\right)
$$

for all sufficiently large $r_{n}$. By the definition of $d_{1}$, for the given $\varepsilon>0$, we have

$$
\left(d_{1}-\varepsilon\right) T(r, f(z)) \leq m\left(r, \frac{1}{f(z)}\right) \leq m\left(r, \frac{1}{f(z+c)}\right)+m\left(r, \frac{f(z+c)}{f(z)}\right)
$$

for all sufficiently large $r$. Then it follows from (2.11) and (2.12) that

$$
\frac{m\left(r_{n}, f(z+c) / f(z)\right)}{T\left(r_{n}, f(z)\right)} \geq \frac{d_{1}-d_{2}-2 \varepsilon}{1+d_{2}+\varepsilon}
$$

for all sufficiently large $r_{n}$. Thus our assertion follows by taking the inferior limit of the left-hand side of the above inequality and then letting $\varepsilon \rightarrow 0$.

We conclude this section by introducing some examples of finite order meromorphic functions having the property $d(0, f(z)) \neq d(0, f(z+c))$ for some constant $c$. The first one is due to Belinskil and Gol'dberg [3] who constructed a meromorphic function with order 1 such that

$$
\begin{aligned}
& d(0, f(z))=1, \quad \text { and } \\
& d(0, f(z+c))=0, \quad \text { for some } c \neq 0 .
\end{aligned}
$$

This example also shows that the finite order case of Valiron's result above is sharp. For the existence of such meromorphic functions, see also [24]. The second one is due to Miles [23] who proved: There exists an entire function $f$ of order $3 / 2<\sigma(f)<\infty$ such that

$$
\begin{aligned}
& d(0, f(z))=0, \quad \text { and } \\
& d(0, f(z+c)) \geq \rho>0, \quad \text { for all } c \neq 0
\end{aligned}
$$

for some $\rho<1$ independent of $c$. For Miles' function, it can actually be shown that $m(r, f(z+c) / f(z)) \neq o(T(r, f))$ for all $r$ in a set $E$ with infinite linear measure [31]. 


\section{Extension of the lemma on the difference quotients}

In this section, we extend the estimate (1.1) to a slightly more general case where the hyper-order of a meromorphic function $f$ is allowed to equal 1 . In another paper [31], two authors of the current paper studied this case using different ideas and showed that the condition $\log T(r, f)=o(r)$ along only one infinite sequence can guarantee that $m(r, f(z+c) / f(z))=o(T(r, f))$ for all $r$ outside a small exceptional set. The method here is due to Halburd, Korhonen and Tohge [19] and for the function in the lemma below we give an estimate with an error term smaller than the one in [31, Theorem 1.4] when $\nu \geq 1$.

Lemma 3.1. Let $f$ be a non-constant meromorphic function. If there exist two positive constants $a$ and $\nu$ such that $\log T(r, f) \leq a r /(\log r)^{2+\nu}$ for all sufficiently large $r$, then for a given small $\varepsilon>0$,

$$
m\left(r, \frac{f(z+c)}{f(z)}\right)=o\left(\frac{T(r, f)}{(\log r)^{\nu-\varepsilon}}\right),
$$

for all $r$ outside of an exceptional set with finite logarithmic measure.

Proof. Without loss of generality, we may suppose that $f(0) \neq 0, \infty$ for otherwise we write $w(z)=z^{k} f(z)$, where $k$ is an integer chosen so that $w(0) \neq 0, \infty$. Let $C>1$ and $r_{0}$ be such that $T(r, f) \geq x_{0}>e$ for all $r \geq r_{0}$. From [7, Lemma 3.3.1] we know that there is a positive, non-decreasing and continuous function $\xi(x), x_{0} \leq x<\infty$, such that the inequality

$$
T\left(r+\frac{r}{\xi(T(r, f))}, f\right) \leq C T(r, f)
$$

holds for all $r$ outside of a set $E$ satisfying

$$
\int_{E \cap\left[r_{0}, R\right]} \frac{d r}{r} \leq \frac{1}{\log C} \int_{x_{0}}^{T(R, f)} \frac{d x}{x \xi(x)}+O(1),
$$

where $R<\infty$. For a given $\varepsilon$ such that $0<2 \varepsilon<\nu$, we choose

$$
\xi(x)=(\log x)(\log \log x)^{1+\varepsilon} .
$$

It follows from (3.2) that the following closed set

$$
E=\left\{r: T\left(r+|c|+\frac{r+|c|}{\xi(T(r+|c|, f))}, f\right) \geq C T(r+|c|, f)\right\}
$$

has finite logarithmic measure. Note that in the above definition we have chosen $\xi(x)$ without separating into the finite order case and the infinite order case of $T(r, f)$ as in (2.8). This may result in an exceptional set a little larger than $E_{1}$ in (2.8) when $f$ is of hyper-order less than 1 , but it makes no difference for our purpose to give an error term small compared to $T(r, f)$. Let

$$
\alpha=1+\frac{1}{\xi(T(r+|c|, f))} .
$$

Then,

$$
T(\alpha(r+|c|), f)=T\left(r+|c|+\frac{r+|c|}{\xi(T(r+|c|, f))}, f\right) \leq C T(r+|c|, f)
$$


for all $r$ outside of the set $E$. By assumption $\log T(r, f) \leq a r /(\log r)^{2+\nu}$ for all $r \geq r_{0}$ and so we have from [19, Lemma 8.2] that

$$
\begin{aligned}
& m\left(r, \frac{f(z+c)}{f(z)}\right)=O\left(\frac{(\log T(r+|c|, f))(\log \log T(r+|c|, f))^{1+\varepsilon}}{\delta(1-\delta) r^{\delta}} T(r+|c|, f)\right) \\
& =O\left(\frac{a(r+|c|)(\log a(r+|c|)-(2+\nu) \log \log (r+|c|))^{1+\varepsilon}}{(\log (r+|c|))^{2+\nu} \delta(1-\delta) r^{\delta}} T(r+|c|, f)\right) .
\end{aligned}
$$

By choosing $h(r)$ in Lemma 3.2 below to be $h(r)=(\log r)^{1+\varepsilon}$, we see that $T(r, f)$ satisfies (3.7) and so we have

$$
T(r+|c|, f)=T(r, f)+o\left(\frac{T(r, f)}{(\log r)^{1+\varepsilon}}\right),
$$

for all $r \rightarrow \infty$ outside of an exceptional set of finite logarithmic measure. Then the estimate (3.1) follows by choosing $\delta=1-1 / \log r$ and combining (3.5) and (3.6) together.

Lemma 3.2. Let $T:[0, \infty) \rightarrow(0, \infty)$ be a non-decreasing continuous function and let $s \in(0, \infty)$. If

$$
\limsup _{r \rightarrow \infty} \frac{h(r) h(r h(r)) \log T(r)}{r}=\zeta
$$

where $\zeta \in[0, \infty)$ and $h:\left[r_{0}, \infty\right) \rightarrow(0, \infty)$ is an increasing function such that

$$
\int_{r_{0}}^{\infty} \frac{1}{t h(t)} d t
$$

converges, then

$$
T(r+s)=T(r)+(\zeta+o(1))\left(\frac{T(r)}{h(r)}\right)
$$

for all $r$ outside of a set $E$ of finite logarithmic measure.

Proof. For a fixed constant $\eta \in \mathbf{R}^{+}$such that $\eta>\zeta$, assume that the set $F_{\eta} \subset[1, \infty)$ defined by

$$
F_{\eta}=\left\{r \in \mathbf{R}^{+}: \frac{T(r+s)-T(r)}{T(r)} \cdot h(r) \geq \eta\right\}
$$

is of infinite logarithmic measure. Note that $F_{\eta}$ is a closed set and therefore it has a smallest element, say $r_{0}$. Set $r_{n}=\min \left(F_{\eta} \cap\left[r_{n-1}+s, \infty\right)\right)$ for all $n \in \mathbf{N}$. Then the sequence $\left\{r_{n}\right\}_{n \in \mathbf{Z}^{+}}$satisfies $r_{n+1}-r_{n} \geq s$ for all $n \in \mathbf{Z}^{+}, F_{\eta} \subset \bigcup_{n=0}^{\infty}\left[r_{n}, r_{n}+s\right]$ and

$$
\left(1+\frac{\eta}{h\left(r_{n}\right)}\right) T\left(r_{n}\right) \leq T\left(r_{n+1}\right)
$$

for all $n \in \mathbf{Z}^{+}$.

Suppose that there exists an $m \in \mathbf{Z}^{+}$such that $r_{n} \geq n h(n)$ for all $r_{n} \geq m$. But then,

$$
\begin{aligned}
\int_{F_{\eta} \cap[1, \infty)} \frac{d t}{t} & \leq \sum_{n=0}^{\infty} \int_{r_{n}}^{r_{n}+s} \frac{d t}{t} \leq \int_{1}^{m} \frac{d t}{t}+\sum_{n=1}^{\infty} \log \left(1+\frac{s}{r_{n}}\right) \\
& \leq \sum_{n=1}^{\infty} \log \left(1+\frac{s}{n h(n)}\right)+O(1)<\infty,
\end{aligned}
$$


which contradicts the assumption $\int_{F_{\eta} \cap[1, \infty)} d t / t=\infty$. Therefore, the sequence $\left\{r_{n}\right\}_{n \in \mathbf{Z}^{+}}$ has a subsequence $\left\{r_{n_{j}}\right\}_{j \in \mathbf{Z}^{+}}$such that $r_{n_{j}}<n_{j} h\left(n_{j}\right)$ for all $j \in \mathbf{Z}^{+}$. Since $r_{n+1}-r_{n} \geq$ $s$, we may assume without loss of generality that $s \geq 1$, by taking another subsequence of $\left\{r_{n_{j}}\right\}_{j \in \mathbf{Z}^{+}}$such that $r_{n_{j+1}}-r_{n_{j}} \geq 1$ if necessary. By iterating (3.10) along the sequence $\left\{r_{n_{j}}\right\}_{j \in \mathbf{Z}^{+}}$, we have

$$
T\left(r_{n_{j}}\right) \geq \prod_{\nu=0}^{n_{j}-1}\left(1+\frac{\eta}{h\left(r_{\nu}\right)}\right) T\left(r_{0}\right)
$$

for all $j \in \mathbf{Z}^{+}$. It follows that

$$
r_{n_{j}} \geq r_{0}+n_{j} s \geq n_{j} s \geq n_{j}
$$

for all $j \in \mathbf{Z}^{+}$, and so

$$
\begin{aligned}
& \limsup _{r \rightarrow \infty} \frac{h(r) h(r h(r)) \log T(r)}{r} \\
& \geq \limsup _{j \rightarrow \infty} \frac{h\left(r_{n_{j}}\right) h\left(r_{n_{j}} h\left(r_{n_{j}}\right)\right)\left(\log T\left(r_{0}\right)+\sum_{\nu=0}^{n_{j}-1} \log \left(1+\frac{\eta}{h\left(r_{\nu}\right)}\right)\right)}{r_{n_{j}}} \\
& \geq \limsup _{j \rightarrow \infty} \frac{h\left(n_{j}\right) h\left(n_{j} h\left(n_{j}\right)\right)\left(\log T\left(r_{0}\right)+n_{j} \log \left(1+\frac{\eta}{h\left(r_{n_{j}}\right)}\right)\right)}{n_{j} h\left(n_{j}\right)} \\
& \left.\geq \limsup _{j \rightarrow \infty} \frac{h\left(n_{j}\right) h\left(n_{j} h\left(n_{j}\right)\right)\left(\log T\left(r_{0}\right)+n_{j} \frac{\eta}{h\left(n_{j} h\left(n_{j}\right)\right)} \log \left(1+\frac{\eta}{h\left(n_{j} h\left(n_{j}\right)\right)}\right)^{\frac{h\left(n_{j} h\left(n_{j}\right)\right)}{\eta}}\right.}{n_{j} h\left(n_{j}\right)}\right) \\
& \geq \limsup _{j \rightarrow \infty} \frac{\eta \cdot n_{j} \cdot(1+o(1)) \cdot h\left(n_{j}\right) h\left(n_{j} h\left(n_{j}\right)\right)}{n_{j} h\left(n_{j}\right) h\left(n_{j} h\left(n_{j}\right)\right)}=\eta,
\end{aligned}
$$

which contradicts (3.7) since we have assumed $\eta>\zeta$. Hence the logarithmic measure of $F_{\eta}$ defined by (3.9) is finite and, since $\eta$ can be arbitrarily chosen, we have

$$
T(r+s)=T(r)+(\zeta+o(1))\left(\frac{T(r)}{h(r)}\right),
$$

for all $r$ outside of a set of finite logarithmic measure. Therefore the assertion (3.8) follows.

Lemma 3.2 is an extension of the growth lemma in [19, Lemma 8.3]. For a small $\varepsilon>0$, we may choose $h(r)=r^{\varepsilon}$ so that the hyper-order of $T(r)$ is less than 1 and get [19, Lemma 8.3]. We remark that the condition in (3.7) cannot be extended to the case of $\zeta=\infty$. This can be seen from the function $T(r)=e^{r}$ which satisfies $T(r+1)=e T(r)$ for all $r \geq 0$.

Note that the relation (2.3) also holds for the counting function $N(r, f)$. By this fact and combining (3.6) with the double inequality (2.2), we immediately get the asymptotic relations

$$
\begin{aligned}
& T(r, f(z+c))=(1+o(1)) T(r, f(z)) \\
& N(r, f(z+c))=(1+o(1)) N(r, f(z))
\end{aligned}
$$


where $r \rightarrow \infty$ outside of an exceptional set of finite logarithmic measure. The relation (3.11) can also be derived from (3.1) and (3.12).

\section{Discrete Borel type growth lemma extensions}

Osgood [27] and, independently, Vojta [29] observed that Nevanlinna's theory of value distribution and Diophantine approximation theory appear to be analogous on a deep level. Based on this observation Vojta composed a "dictionary" between the two theories. In this dictionary Nevanlinna theory appears to be ahead of Diophantine approximation theory in the sense that deep open conjectures in Diophantine approximation correspond to known classical results in Nevanlinna theory. Despite of the highly promising formal connections between these two theories, there are not many new results in Diophantine approximation which were born as a result of using a proof from Nevanlinna theory with concepts from number theory. The main reason for this is the lack of an analogue for the derivative in Vojta's dictionary. Most classical results in Nevanlinna theory rely - one way or another - on the concept of derivative in their proofs.

By replacing the continuous variable $r$ in Lemma 3.2 with a sequence of positive numbers, we have the following discrete analogue of Lemma 3.2. The method of proof of Lemma 3.2 does not rely on derivatives, which enables us to use a discrete version of its proof. Our result is an extension of [2, Lemma 8] due to Al-Ghassani and Halburd, who applied their result to consider non-linear discrete equations with solutions $y_{n} \in \mathbf{Q}$ having slow height growth in terms of $n$.

For clarity, in what follows we use $h(n)=h_{n}$ for a discrete sequence $\left\{h_{n}\right\}$.

Lemma 4.1. Let $\left\{T_{n}\right\}_{n \geq n_{0}}\left(n_{0}>0\right)$ be a non-decreasing sequence of positive numbers and let $s$ be a fixed positive integer. If

$$
\limsup _{n \rightarrow \infty} \frac{h(n) h(n h(n)) \log T_{n}}{n}=\zeta,
$$

where $\zeta \in[0, \infty)$ and $h(n)$ is an increasing sequence of positive numbers such that

$$
\sum_{n=n_{0}}^{\infty} \frac{1}{n h(n)}<+\infty
$$

then

$$
T_{n+s}=T_{n}+(\zeta+o(1))\left(\frac{T_{n}}{h(n)}\right)
$$

for all $n$ outside of a set $E$ of finite discrete logarithmic measure, i.e. $\sum_{n \in E} 1 / n<\infty$.

Proof. For a fixed constant $\eta \in \mathbf{R}^{+}$such that $\eta>\zeta$, assume that the set $F_{\eta} \subset \mathbf{N}$ defined by

$$
F_{\eta}=\left\{n \geq n_{0}: \frac{T_{n+s}-T_{n}}{T_{n}} \cdot h(n) \geq \eta\right\}
$$

is of infinite discrete logarithmic measure, i.e. $\sum_{n \in F_{\eta}} 1 / n=\infty$. Let $r_{0}=\min \left(F_{\eta}\right)$ and, for all $n \in \mathbf{N}$, set $r_{n}=\min \left(F_{\eta} \cap\left[r_{n-1}+s, \infty\right)\right)$. Then the sequence $\left\{r_{n}\right\}_{n \in \mathbf{N}}$ satisfies $r_{n+1}-r_{n} \geq s$ for all $n \in \mathbf{Z}^{+}, F_{\eta} \subset \bigcup_{n=0}^{\infty}\left[r_{n}, r_{n}+s\right]$ and

$$
\left(1+\frac{\eta}{h\left(r_{n}\right)}\right) T_{r_{n}} \leq T_{r_{n}+s} \leq T_{r_{n+1}}
$$


for all $n \in \mathbf{Z}^{+}$. Suppose that there exists an integer $m \in \mathbf{Z}^{+}$such that $r_{n} \geq n h(n)$ for all $n \geq m$. But then, there is a positive constant $S$ such that

$$
\begin{aligned}
\sum_{j \in F_{\eta}} \frac{1}{j} & \leq S+\sum_{n=m}^{\infty} \sum_{k=[n h(n)]}^{[n h(n)]+s} \frac{1}{k} \leq S+\sum_{n=m}^{\infty} \int_{[n h(n)]-1}^{[n h(n)]+s} \frac{1}{t} d t \leq S+\sum_{n=m}^{\infty} \int_{n h(n)-2}^{n h(n)+s} \frac{1}{t} d t \\
& \leq S+\sum_{n=m}^{\infty} \ln \left(1+\frac{s+2}{n h(n)-2}\right) \leq S+\sum_{n=m}^{\infty} \frac{s+2}{n h(n)-2}<\infty
\end{aligned}
$$

where $[n h(n)]$ denotes the largest integer not exceeding $n h(n)$, which is a contradiction with the assumption $\sum_{j \in F_{\eta}} 1 / j=\infty$. Therefore, the sequence $\left\{r_{n}\right\}_{n \in \mathbf{N}}$ has a subsequence $\left\{r_{n_{j}}\right\}_{j \in \mathbf{Z}^{+}}$such that $r_{n_{j}} \leq n_{j} h\left(n_{j}\right)$ for all $j \in \mathbf{Z}^{+}$. Then, as in the proof of Lemma 3.2, by iterating (4.4) along the sequence $\left\{r_{n_{j}}\right\}_{j \in \mathbf{Z}^{+}}$, it can be shown that $r_{n_{j}} \geq n_{j}$ for all $j \in \mathbf{Z}^{+}$and that the superior limit of $h(n) h(n h(n)) \log T_{n} / n$ along the sequence $r_{n_{j}}$ is $\geq \eta$, which yields a contradiction to (4.1). We omit those details. This implies that the discrete logarithmic measure of $F_{\eta}$ defined by (4.3) must be finite, and so

$$
T_{n+s}=T_{n}+(\zeta+o(1))\left(\frac{T_{n}}{h(n)}\right)
$$

for all $n$ outside of a set of finite discrete logarithmic measure. Thus the assertion (4.2) follows.

Analogous to the continuous case, we take $h(n)$ in Lemma 4.1 to be $h(n)=$ $(\log n)^{1+\varepsilon}$, where $\varepsilon>0, n \geq n_{0}>e$. Let $a, \nu$ be two positive numbers and $\left\{T_{n}\right\}_{n \geq n_{0}}$ a sequence of $n$ such that $\log T_{n} \leq a n /(\log n)^{2+\nu}$ for all sufficiently large $n$. Then $\left\{T_{n}\right\}_{n \geq n_{0}}$ satisfies the condition (4.1) and so by Lemma 4.1 we have

$$
T_{n+s}=T_{n}+o\left(\frac{T_{n}}{(\log n)^{1+\varepsilon}}\right)
$$

where $n \rightarrow \infty$ outside of a set of finite discrete logarithmic measure. Also, the function $T_{n}=\exp \left(e^{n}\right)$ shows that $\zeta$ in Lemma 4.1 cannot be extended to the case of $\zeta=\infty$.

\section{Malmquist's theorem for discrete equations}

The algebraic entropy $[4,20]$ of a discrete equation is defined as

$$
\lim _{j \rightarrow \infty} \frac{\log d_{j}}{j}
$$

where $d_{j}$ is the degree of the $j^{\text {th }}$ iterate of a discrete equation as a rational function of its initial conditions. If the algebraic entropy of a discrete equation is zero, then this is considered to be a strong sign of integrability of the equation. Consider as an example discrete equation

$$
y_{n+1}=R\left(n, y_{n}\right)=\frac{P\left(n, y_{n}\right)}{Q\left(n, y_{n}\right)},
$$

where $P\left(n, y_{n}\right)$ and $Q\left(n, y_{n}\right)$ are coprime polynomials in $y_{n}$ having rational coefficients in $\mathbf{Q}[n]$. For the autonomous version of (5.1), we have $d_{j}=\left[\operatorname{deg}_{y_{0}}(R)\right]^{j}$ and so the algebraic entropy is equal to $\log \operatorname{deg}_{y_{0}}(R)$ in this case. This implies that the algebraic entropy of (5.1) is zero if and only if (5.1) is the discrete Riccati equation. For a review on applications of algebraic entropy to the second order discrete equations, see [14]. 
Halburd [15] has shown, assuming that the heights of the coefficients are small compared to the height of the solution, that the heights of iterates of the discrete equation (5.1) over number fields grow exponentially, unless $\operatorname{deg}_{y_{0}}(R)=1$. Using this idea of Diophantine integrability, Al-Ghassani and Halburd obtained an extension of this result to the second order case by singling out the discrete Painlevé II equation [2].

As the final result of this study, we will apply Lemma 4.1 to give an improvement of Halburd's result on the first order discrete equations. Before stating the result, we need one more definition. Let $k$ be a number field, and let $\left\{y_{n}\right\}_{n \in \mathbf{N}} \subset k$ be a solution of (5.1), where the coefficients are in $k[n]$. For $x \in k$ we denote by $H(x)$ the height and by $h(x)=\log H(x)$ the logarithmic height of $x$. We say that $\left\{y_{n}\right\}_{n \in \mathbf{N}}$ is admissible if the logarithmic heights of all coefficients of (5.1) are of the growth $o\left(h\left(y_{n}\right)\right)$ as $n \rightarrow \infty$ outside of an exceptional set of finite discrete logarithmic measure $\sum_{n \in E} 1 / n<\infty$. This definition is an exact Diophantine analogue of the notion of admissible meromorphic solution of a difference equation in the spirit of Vojta's dictionary [29].

Theorem 5.1. Let $k$ be a number field, and let $\left\{y_{n}\right\}_{n \in \mathbf{N}} \subset k$ be an admissible solution of (5.1), where the coefficients are in $k[n]$. If

$$
\limsup _{n \rightarrow \infty} \frac{\log \sum_{k=1}^{n} h\left(y_{k}\right)}{n /(\log n)^{2+\nu}}=0
$$

for some $\nu>0$, then $\operatorname{deg}_{y_{0}}(R)=1$.

Proof. By taking the logarithmic height of both sides of (5.1), it follows that

$$
h\left(y_{n+1}\right)=\operatorname{deg}_{y_{0}}(R) h\left(y_{n}\right)+o\left(h\left(y_{n}\right)\right)
$$

as $n \rightarrow \infty$ outside of an exceptional set $E$ of finite discrete logarithmic measure. Therefore,

$$
\sum_{k=1}^{n+1} h\left(y_{k}\right)=\operatorname{deg}_{y_{0}}(R) \sum_{k=1}^{n} h\left(y_{k}\right)+o\left(h\left(y_{n}\right)\right),
$$

and so, by applying Lemma 4.1 with $T_{n}=\sum_{k=1}^{n} h\left(y_{k}\right)$, we have the assertion.

\section{References}

[1] Ablowitz, M. J., R. G. Halburd, and B. Herbst: On the extension of the Painlevé property to difference equations. - Nonlinearity 13, 2000, 889-905.

[2] Al-Ghassani, A., and R. G. Halburd: Height growth of solutions and a discrete Painlevé equation. - Nonlinearity 28:7, 2015, 2379-2396.

[3] BeLinskil̆, P. P., and A. A. Gol' DBerg: Application of a theorem on conformal mappings to questions of invariance of defects of meromorphic functions. - Ukrain. Mat. Ž. 6, 1954, 263-269.

[4] Bellon, M. P., and C.-M. Viallet: Algebraic entropy. - Comm. Math. Phys. 204, 1999, $425-437$.

[5] Bergweiler, W., and J. K. Langley: Zeros of differences of meromorphic functions. - Math. Proc. Cambridge Philos. Soc. 142:1, 2007, 133-147.

[6] Cheng, K. H., and Y. M. Chinng: Nevanlinna theory of the Wilson divided-difference operator. - Ann. Acad. Sci. Fenn. Math. 42:1, 2017, 175-209.

[7] Cherry, W., and Z. Ye: Nevanlinna's theory of value distribution. - Springer-Verlag, Berlin, 2001.

[8] Chiang, Y. M., and S. J. Feng: On the Nevanlinna characteristic of $f(z+\eta)$ and difference equations in the complex plane. - Ramanujan J. 16:1, 2008, 105-129. 
[9] Chiang, Y. M., and S. J. Feng: On the growth of logarithmic differences, difference quotients and logarithmic derivatives of meromorphic functions. - Trans. Amer. Math. Soc. 361:7, 2009, $3767-3791$.

[10] Chinng, Y.M., and S. J. Feng: On the growth of logarithmic difference of meromorphic functions and a Wiman-Valiron estimate. - Constr. Approx. 44:3, 2016, 313-326.

[11] Chinng,Y. M., and S. J. Feng: Nevanlinna theory of the Askey-Wilson divided difference operator. - Adv. Math. 329, 2018, 217-272.

[12] Chinng, Y. M., and X. D. LuO: Difference Nevanlinna theories with vanishing and infinite periods. - Michigan Math. J. 66:3, 2017, 451-480.

[13] Gol'dberg, A. A., and I. V. Ostrovskǐ̆: Value distribution of meromorphic functions. Transl. Math. Monogr. 236, Amer. Math. Soc., Providence, RI, 2008.

[14] Grammaticos, B., R. G. Halburd, A. Ramani, and C.-M. Viallet: How to detect the integrability of discrete systems. - J. Phys. A 42:45, 2009, 454002, 30.

[15] Halburd, R. G.: Diophantine integrability. - J. Phys. A: Math. Gen. 38, 2005, L263-L269.

[16] Halburd, R. G., and R. J. Korhonen: Difference analogue of the lemma on the logarithmic derivative with applications to difference equations. - J. Math. Anal. Appl. 314:2, 2006, 477487.

[17] Halburd, R. G., and R. J. Korhonen: Nevanlinna theory for the difference operator. - Ann. Acad. Sci. Fenn. Math. 31:2, 2006, 463-478.

[18] Halburd, R. G., and R. J. Korhonen: Nondecreasing functions, exceptional sets and generalized Borel lemmas. - J. Aust. Math. Soc. 88:3, 2010, 353-361.

[19] Halburd, R. G., R. J. Korhonen, and K. Tohge: Holomorphic curves with shift-invariant hyperplane preimages. - Trans. Amer. Math. Soc. 366:8, 2014, 4267-4298.

[20] Hietarinta, J., and C.-M. Viallet: Singularity confinement and chaos in discrete systems. - Phys. Rev. Lett. 81, 1998, 325-328.

[21] IshizAKI, K., and N. YANAGiHARA: Wiman-Valiron method for difference equations. - Nagoya Math. J. 175, 2004, 75-102.

[22] LAINE, I.: Nevanlinna theory and complex differential equations. - de Gruyter Stud. Math. 15, Walter de Gruyter \& Co., Berlin, 1993.

[23] Miles, J.: Some examples of the dependence of the Nevanlinna deficiency upon the choice of origin. - Proc. London Math. Soc. (3) 47:1, 1983, 145-176.

[24] Mori, S.: An example on defect of a composite function. - Tohoku Math. J. (2) 24, 1972, 269-274.

[25] Nevanlinna, R.: Zur Theorie der meromorphen Funktionen. - Acta Math. 46, 1925, 1-99.

[26] Nevanlinna, R.: Analytic functions. - Grundlehren Math. Wiss. 162, Springer-Verlag, New York, 1970.

[27] Osgood, C. F.: Sometimes effective Thue-Siegel-Roth-Schmidt-Nevanlinna bounds, or better. - J. Number Theory 21:3, 1985, 347-389.

[28] Valiron, G.: Valeurs exceptionnelles et valeurs déficientes des fonctions méromorphes. - C. R. Acad. Sci. Paris 225, 1947, 556-558.

[29] Vojta, P.: Diophantine approximations and value distribution theory. - Lecture Notes in Math. 1239, Springer-Verlag, Berlin, 1987.

[30] Yanagihara, N.: Meromorphic solutions of some difference equations. - Funkcial. Ekvac. 23:3, 1980, 309-326.

[31] Zheng, J. H., and R. Korhonen: Studies of differences from the point of view of Nevanlinna theory. - https://arxiv.org/abs/1806.00212. 